\title{
Compressive STEM-EELS
}

\author{
Andrew Stevens ${ }^{1,2}$, Libor Kovarik ${ }^{1}$, Hao Yang ${ }^{3}$, Yunchen $\mathrm{Pu}^{2}$, Lawrence Carin ${ }^{2}$, and Nigel D. Browning ${ }^{1}$ \\ 1. Pacific Northwest National Laboratory, Richland USA \\ 2. Duke University, ECE, Durham USA \\ 3. Lawrence Berkeley National Laboratory, Berkeley USA
}

The collection of electron energy loss spectra (EELS) via scanning transmission electron microscopy (STEM) generally requires a specimen to withstand a large radiation dose. Moreover, significant drift can occur while the spectra are collected. Recent advances in electron microscopy have shown that a data reduction of up to $90 \%$ is possible for $\mathrm{HAADF} / \mathrm{ABF}$ imaging and TEM video [1, 2, 3]. These advances depend on the mathematical theory of compressive sensing (CS) [4]. For STEM [1], the method in [5] (BPFA) was used for a special case of CS called inpainting; the pixels of the image were missing at random. The goal of the inpainting task is to fill-in missing pixels.

Naively, the inpainting approach can be extended to hyperspectral images (e.g., STEM-EELS data) by extending the missing pixels through all of the spectral bins (i.e., removing entire spectra at random spatial locations). However, it has been shown that incorporating spectral information can improve results in infrared hyperspectral imaging [6].

We have developed a new reconstruction algorithm for CS that respects spatial-spectral-temporal structure in any number of dimensions [7]. Our algorithm can be used for denoising, inpainting, CS, and image classification. Spatial-spectral structure is especially important in hyperspectral CS. Previous algorithms ignore this structure and instead compute solutions assuming every pixel (voxel) is independent.

We also propose the first CS approach for STEM-EELS (that improves when our new algorithm is used). Instead of masking spatial locations, we mask $50 \%$ of the spectral bins and sum multiple masked spectra into a single measurement. A diagram of the process is shown in figure 1. Because this approach takes multiplexed measurements, it is more resilient to noise, thus dwell time could be decreased. Moreover, this approach can be combined with inpainting for further dose reduction and speed improvements.

Figure 2 shows an example of our CS approach using data from a Pr-doped STO $\Sigma 13$ grain boundary sample collected on an ARM200. The coded measurements are missing 50\% of the spectral information. The missing spectral bins are chosen randomly. The compressed measurement is the sum of the coded measurements. We compressed the entire hyperspectral image in this manner, using $2 \times 2$ blocks. The algorithm only used the compressed data. We can see that the reconstructions have less noise, and the spectral peaks are maintained. Our approach would decrease acquisition time by $4 \times$ on this example [8]. 
References:

[1] A Stevens, H Yang, L Carin et al. Microscopy 63(1), (2014), pp. 41.

[2] A Stevens, L Kovarik, P Abellan et al. Advanced Structural and Chemical Imaging 1(1), (2015), pp. 1.

[3] A Stevens, L Kovarik, P Abellan et al. Microscopy and Microanalysis 21(S3), (2015), pp. 1583.

[4] RG Baraniuk. IEEE signal processing magazine 24(4).

[5] M Zhou, H Chen, J Paisley et al. Image Processing, IEEE Transactions on 21(1), (2012), pp. 130.

[6] Z Xing, M Zhou, A Castrodad et al. SIAM Journal on Imaging Sciences 5(1), (2012), pp. 33.

[7] A Stevens, Y Pu, Y Sun et al. Submitted .

[8] This work was supported in part by US DOE Grant No. DE-FG02-03ER46057. This research is also part of the Chemical Imaging Initiative conducted under the LDRD Program at PNNL. PNNL is a multi-program lab operated by Battelle Memorial Inst. for the US DOE; contract DE-AC05-76RL01830.
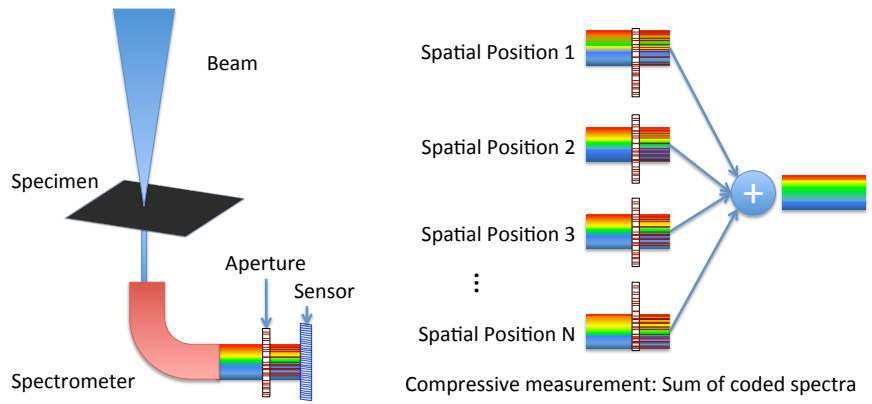

Figure 1: Diagram of the compression process for Compressive EELS.
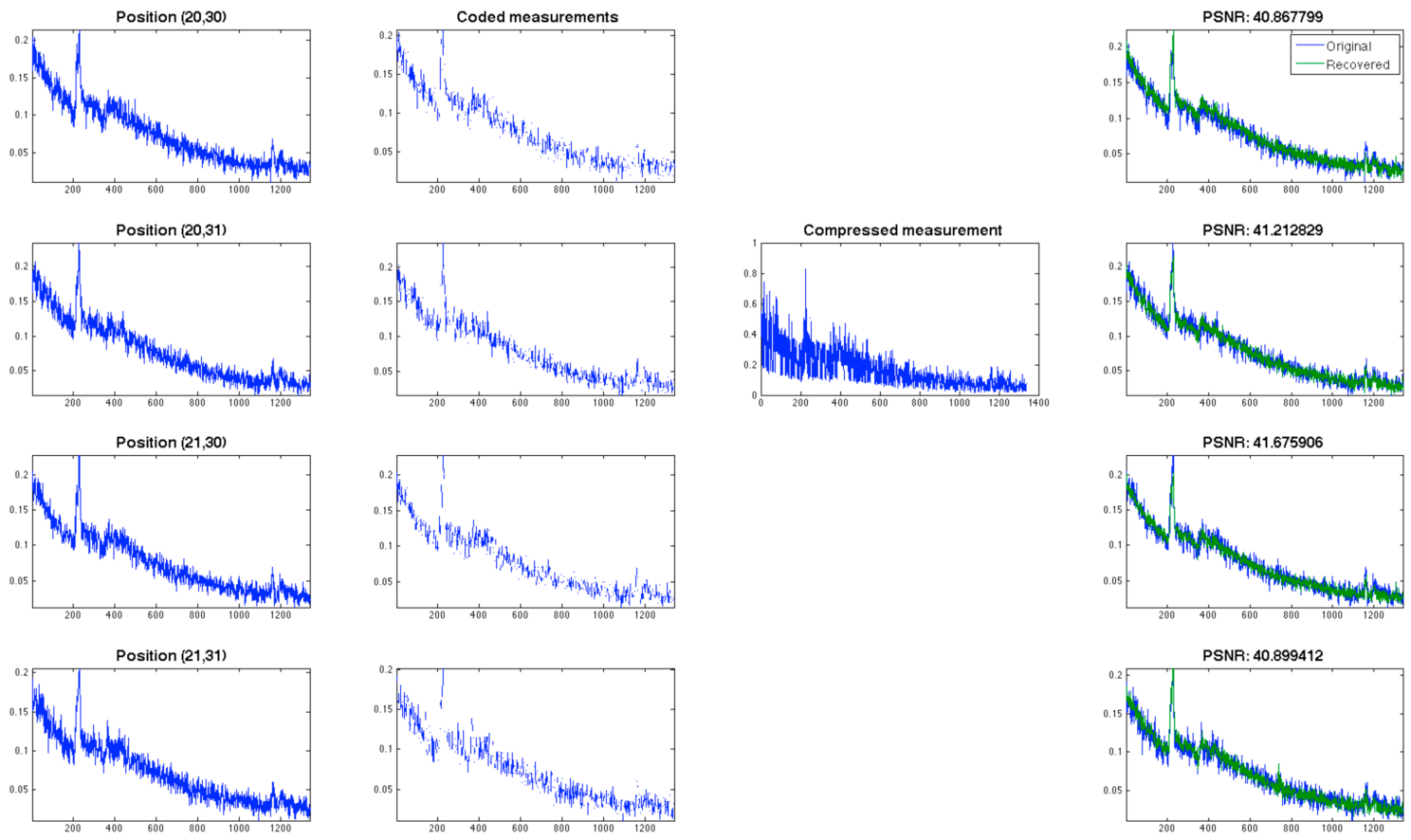

Figure 2: Simulated example of Compressive EELS. From left to right: spectra, coded spectra, compressed measurement, and reconstructed spectra overlaid on original spectral. The noise is significantly reduced in the reconstruction and the spectral peaks are maintained (including two small peaks on the right). 